\title{
A Generalized Nonlinear Random Equations with Random Fuzzy Mappings in Uniformly Smooth Banach Spaces
}

\author{
Nawitcha Onjai-Uea ${ }^{1,2}$ and Poom Kumam ${ }^{1,2}$ \\ ${ }^{1}$ Department of Mathematics, Faculty of Science, King Mongkut's University of \\ Technology Thonburi (KMUTT), Bangmod, Bangkok 10140, Thailand \\ ${ }^{2}$ Centre of Excellence in Mathematics, CHE, Sriayudthaya Road, Bangkok 10400, Thailand \\ Correspondence should be addressed to Poom Kumam, poom.kum@kmutt.ac.th \\ Received 26 July 2010; Accepted 31 October 2010 \\ Academic Editor: Yeol J. E. Cho
}

Copyright (C) 2010 N. Onjai-Uea and P. Kumam. This is an open access article distributed under the Creative Commons Attribution License, which permits unrestricted use, distribution, and reproduction in any medium, provided the original work is properly cited.

\begin{abstract}
We introduce and study the general nonlinear random $(H, \eta)$-accretive equations with random fuzzy mappings. By using the resolvent technique for the $(H, \eta)$-accretive operators, we prove the existence theorems and convergence theorems of the generalized random iterative algorithm for this nonlinear random equations with random fuzzy mappings in $q$-uniformly smooth Banach spaces. Our result in this paper improves and generalizes some known corresponding results in the literature.
\end{abstract}

\section{Introduction}

Fuzzy Set Theory was formalised by Professor Lofti Zadeh at the University of California in 1965 with a view to reconcile mathematical modeling and human knowledge in the engineering sciences. The concept of fuzzy sets is incredible wide range of areas, from mathematics and logics to traditional and advanced engineering methodologies. Applications are found in many contexts, from medicine to finance, from human factors to consumer products, and from vehicle control to computational linguistics.

Random variational inequality theories is an important part of random function analysis. These topics have attracted many scholars and exports due to the extensive applications of the random problems (see, e.g., [1-17]). In 1997, Huang [3] first introduced the concept of random fuzzy mapping and studied the random nonlinear quasicomplementarity problem for random fuzzy mappings. Further, Huang studied the random generalized nonlinear variational inclusions for random fuzzy mappings in Hilbert spaces. Ahmad and Bazán [18] 
studied a class of random generalized nonlinear mixed variational inclusions for random fuzzy mappings and constructed an iterative algorithm for solving such random problems.

Very recently, Lan et al. [11], introduced and studied a class of general nonlinear random multivalued operator equations involving generalized $m$-accretive mappings in Banach spaces and an iterative algorithm with errors for this nonlinear random multivalued operator equations.

Inspired and motivated by recent works in these fields (see $[2,13,14,18-29]$ ), in this paper, we introduce and study a class of general nonlinear random equations with random fuzzy mappings in Banach spaces. By using Chang's lemma and the resolvent operator technique for $(H, \eta)$-accretive mapping. We prove the existence and convergence theorems of the generalized random iterative algorithm for this nonlinear random equations with random fuzzy mappings in $q$-uniformly smooth Banach spaces. Our results improve and extend the corresponding results of recent works.

\section{Preliminaries}

Throughout this paper, let $(\Omega, \mathcal{A}, \mu)$ be a complete $\sigma$-finite measure space and $E$ be a separable real Banach space. We denote by $\mathcal{B}(E),(\cdot, \cdot)$ and $\|\cdot\|$ the class of Borel $\sigma$-fields in $E$, the inner product and the norm on $E$, respectively. In the sequel, we denote $2^{E}, \mathrm{CB}(E)$ and $\widehat{H}$ by $2^{E}=$ $\{A: A \in E\}, \mathrm{CB}(E)=\{A \subset E: A$ is nonempty, bounded and closed $\}$ and the Hausdorff metric on $\mathrm{CB}(E)$, respectively.

Next, we will use the following definitions and lemmas.

Definition 2.1. An operator $x: \Omega \rightarrow E$ is said to be measurable if, for any $B \in B(E),\{t \in \Omega$ : $x(t) \in B\} \in \mathcal{A}$.

Definition 2.2. A operator $F: \Omega \times E \rightarrow E$ is called a random operator if for any $x \in E, F(t, x)=$ $y(t)$ is measurable. A random operator $F$ is said to be continuous (resp. linear, bounded) if for any $t \in \Omega$, the operator $F(t, \cdot): E \rightarrow E$ is continuous (resp. linear, bounded).

Similarly, we can define a random operator $a: \Omega \times E \times E \rightarrow E$. We will write $F_{t}(x)=$ $F(t, x(t))$ and $a_{t}(x, y)=a(t, x(t), y(t))$ for all $t \in \Omega$ and $x(t), y(t) \in E$.

It is well known that a measurable operator is necessarily a random operator.

Definition 2.3. A multivalued operator $G: \Omega \rightarrow 2^{E}$ is said to be measurable if, for any $B \in$ $B(E), G^{-1}(B)=\{t \in \Omega: G(t) \cap B \neq \emptyset\} \in \mathscr{A}$.

Definition 2.4. A operator $u: \Omega \rightarrow E$ is called a measurable selection of a multivalued measurable operator $\Gamma: \Omega \rightarrow 2^{E}$ if $u$ is measurable and for any $t \in \Omega, u(t) \in \Gamma(t)$.

Lemma 2.5 (see [19]). Let $M: \Omega \times E \rightarrow C B(E)$ be a $H$-continuous random multivalued operator. Then, for any measurable operator $x: \Omega \rightarrow E$, the multivalued operator $M(\cdot, x(\cdot)): \Omega \rightarrow C B(E)$ is measurable.

Lemma 2.6 (see [19]). Let $M, V: \Omega \times E \rightarrow C B(E)$ be two measurable multivalued operators, $\epsilon>0$ be a constant and $x: \Omega \rightarrow E$ be a measurable selection of $M$. Then there exists a measurable selection $y: \Omega \rightarrow E$ of $V$ such that, for any $t \in \Omega$,

$$
\|x(t)-y(t)\| \leq(1+\epsilon) \widehat{H}(M(t), V(t)) .
$$


Definition 2.7. A multivalued operator $F: \Omega \times E \rightarrow 2^{E}$ is called a random multivalued operator if, for any $x \in E, F(\cdot, x)$ is measurable. A random multivalued operator $F: \Omega \times E \rightarrow \mathrm{CB}(E)$ is said to be $H$-continuous if, for any $t \in \Omega, F(t, \cdot)$ is continuous in $\widehat{H}(\cdot, \cdot)$, where $\widehat{H}(\cdot, \cdot)$ is the Hausdorff metric on $\mathrm{CB}(E)$ defined as follows: for any given $A, B \in \mathrm{CB}(E)$,

$$
\widehat{H}(A, B)=\max \left\{\sup _{x \in A} \inf _{y \in B} d(x, y), \sup _{y \in B} \inf _{x \in A} d(x, y)\right\} .
$$

Let $\mathcal{F}(E)$ be the family of all fuzzy sets over $E$. A mapping $F: E \rightarrow \mathcal{F}(E)$ is called a fuzzy mapping over $E$.

If $F$ is a fuzzy mapping over $E$, then $F(x)$ (denoted by $F_{x}$ ) is fuzzy set on $E$, and $F_{x}(y)$ is the membership degree of the point $y$ in $F_{x}$. Let $A \in \mathcal{F}(E), \alpha \in[0,1]$. Then the set

$$
(A)_{\alpha}=\{x \in E: A(x) \geq \alpha\}
$$

is called a $\alpha$-cut set of fuzzy set $A$.

(i) A fuzzy mapping $F: \Omega \rightarrow \mathcal{F}(E)$ is called measurable if, for any given $\alpha \in(0,1]$, $(F(\cdot))_{\alpha}: \Omega \rightarrow 2^{E}$ is a measurable multivalued mapping.

(ii) A fuzzy mapping $F: \Omega \times E \rightarrow \mathcal{F}(E)$ is called a random fuzzy mapping if, for any $x \in E, F(\cdot, x): \Omega \rightarrow \mathcal{F}(E)$ is a measurable fuzzy mapping.

Let $K, T, G: \Omega \times E \rightarrow \mathcal{F}(E)$ be three random fuzzy mappings satisfying the following condition $(\mathrm{C})$ : there exists three mappings $a, b, c: E \rightarrow(0,1]$, such that

$$
\left(K_{t, x}\right)_{a(x)} \in \mathrm{CB}(E), \quad\left(T_{t, x}\right)_{b(x)} \in \mathrm{CB}(E), \quad\left(G_{t, x}\right)_{c(x)} \in \mathrm{CB}(E), \quad \forall(t, x) \in \Omega \times E .
$$

By using the random fuzzy mappings $K, T$ and $G$, we can define the three multivalued mappings $\tilde{K}, \tilde{T}$ and $\tilde{G}$ as follows, respectively.

$$
\begin{array}{cc}
\tilde{K}: \Omega \times E \longrightarrow \mathrm{CB}(E), \quad(t, x) \longrightarrow\left(K_{t, x}\right)_{a(x)}, \quad \forall(t, x) \in \Omega \times E, \\
\tilde{T}: \Omega \times E \longrightarrow \mathrm{CB}(E), \quad(t, x) \longrightarrow\left(T_{t, x}\right)_{b(x)}, \quad \forall(t, x) \in \Omega \times E, \\
\tilde{G}: \Omega \times E \longrightarrow \mathrm{CB}(E), \quad(t, x) \longrightarrow\left(G_{t, x}\right)_{c(x)}, \quad \forall(t, x) \in \Omega \times E .
\end{array}
$$

It means that

$$
\begin{gathered}
\tilde{K}(t, x)=\left(K_{t, x}\right)_{a(x)}=\left\{z \in E,\left(K_{t, x}\right)(z) \geq a(x)\right\} \in \mathrm{CB}(E), \\
\tilde{T}(t, x)=\left(T_{t, x}\right)_{b(x)}=\left\{z \in E,\left(T_{t, x}\right)(z) \geq b(x)\right\} \in \mathrm{CB}(E), \\
\tilde{G}(t, x)=\left(G_{t, x}\right)_{c(x)}=\left\{z \in E,\left(G_{t, x}\right)(z) \geq c(x)\right\} \in \mathrm{CB}(E) .
\end{gathered}
$$

It easy to see that $\tilde{K}, \widetilde{T}$ and $\widetilde{G}$ are the random multivalued mappings. We call $\tilde{K}, \widetilde{T}$ and $\widetilde{G}$ are random multivalued mappings induced by fuzzy mappings $K, T$ and $G$, respectively. 
Suppose that $p, S: \Omega \times E \rightarrow E$ and $M: \Omega \times E \times E \rightarrow 2^{E}$ with $\operatorname{Im}(p) \cap \operatorname{dom}(M(t, \cdot, s)) \neq \emptyset$, $H: \Omega \times E \rightarrow E$ and $N: \Omega \times E \times E \times E \rightarrow E$ be two single-valued mappings. Let $K, T, G$ : $\Omega \times E \rightarrow \mathcal{F}(E)$ be three random fuzzy mappings satisfying the condition $(C)$. Given mappings $a, b, c: E \rightarrow(0,1]$. Now, we consider the following problem:

Find measurable mappings $x, u, v, w: \Omega \rightarrow E$ such that for all $t \in \Omega, x(t) \in E$, $K_{t, x(t)}(u(t)) \geq a(x(t)), T_{t, x(t)}(v(t)) \geq b(x(t)), G_{t, x(t)}(w(t)) \geq c(x(t))$ and

$$
0 \in N(t, S(t, x(t)), u(t), v(t))+M(t, p(t, x(t)), w(t)) .
$$

The problem (2.7) is called random variational inclusion problem for random fuzzy mappings in Banach spaces. The set of measurable mappings $(x, u, v, w)$ is called a random solution of (2.7).

Some special cases of (2.7):

(1) If $G$ is a single-valued operator, $p \equiv I$, where $I$ is the identity mapping and $N(t, x, y, z)=f(t, z)+g(t, x, y)$ for all $t \in \Omega$ and $x, y, z \in E$, then (2.7) is equivalent to finding $x, v: \Omega \rightarrow E$ such that $v(t) \in T(t, x(t))$ and

$$
0 \in f(t, v(t))+g(t, S(t, x(t)), u(t))+M(t, x(t), G(t, x(t))),
$$

for all $t \in \Omega$ and $u \in M(t, x(t))$. The problem (2.8) was considered and studied by Agarwal et al. [1], when $G \equiv I$.

If $M(t, x, s)=M(t, x)$ for all $t \in \Omega, x, s \in E$ and, for all $t \in \Omega, M(t, \cdot): E \rightarrow 2^{E}$ is a $\left(H_{t}, \eta\right)$-accretive mapping, then (2.7) reduces to the following generalized nonlinear random multivalued operator equation involving $\left(H_{t}, \eta\right)$-accretive mapping in Banach spaces:

Find $x, v: \Omega \rightarrow E$ such that $v(t) \in T(t, x(t))$ and

$$
0 \in N(t, S(t, x(t)), u(t), v(t))+M(t, g(t, x(t))),
$$

for all $t \in \Omega$ and $u(t) \in M(t, x(t))$.

The generalized duality mapping $J_{q}: E \rightarrow 2^{E^{*}}$ is defined by

$$
J_{q}(x)=\left\{f^{*} \in E^{*}:\left\langle x, f^{*}\right\rangle=\|x\|^{q},\left\|f^{*}\right\|=\|x\|^{q-1}\right\},
$$

for all $x \in E$, where $q>1$ is a constant. In particular, $J_{2}$ is the usual normalized duality mapping. It is well known that, in general, $J_{q}(x)=\|x\|^{q-2} J_{2}(x)$ for all $x \neq 0$ and $J_{q}$ is singlevalued if $E^{*}$ is strictly convex (see, e.g., [29]). If $E=H$ is a Hilbert space, then $J_{2}$ becomes the identity mapping of $H$. In what follows we will denote the single-valued generalized duality mapping by $j_{q}$.

The modules of smoothness of $E$ is the function $\rho_{E}:[0, \infty) \rightarrow[0, \infty)$ defined by

$$
\rho_{E}(t)=\sup \left\{\frac{1}{2}\|x+y\|+\|x-y\|-1:\|x\| \leq 1,\|y\| \leq t\right\}
$$

A Banach space $E$ is called uniformly smooth if $\lim _{t \rightarrow 0}\left(\rho_{E}(t) / t\right)=0$ and $E$ is called $q$-uniformly smooth if there exists a constant $c>0$ such that $\rho_{E(t)} \leq c t^{q}$, where $q>1$ is a real number. 
It is well known that Hilbert spaces, $L_{p}\left(\right.$ or $\left.l_{p}\right)$ spaces, $1<p<\infty$ and the Sobolev spaces $W^{m, p}, 1<p<\infty$, are all $q$-uniformly smooth.

In the study of characteristic inequalities in a $q$-uniformly smooth Banach space, $\mathrm{Xu}$ [30] proved the following result.

Lemma 2.8. Let $q>1$ be a given real number and $E$ be a real uniformly smooth Banach space. Then $E$ is $q$-uniformly smooth if and only if there exists a constant $c_{q}>0$ such that, for all $x, y \in E$ and $j_{q}(x) \in J_{q}(x)$, the following inequality holds:

$$
\|x+y\|^{q} \leq\|x\|^{q}+q\left\langle y, j_{q}(x)\right\rangle+c_{q}\|y\|^{q} .
$$

Definition 2.9. A random operator $p: \Omega \times E \rightarrow E$ is said to be:

(a) $\alpha$-strongly accretive if there exists $j_{2}(x(t)-y(t)) \in J_{2}(x(t)-y(t))$ such that

$$
\left\langle g_{t}(x)-g_{t}(y), j_{2}(x(t)-y(t))\right\rangle \geq \alpha(t)\|x(t)-y(t)\|^{2},
$$

for all $x(t), y(t) \in E$ and $t \in \Omega$, where $\alpha(t)>0$ is a real-valued random variable;

(b) $\beta$-Lipschitz continuous if there exists a real-valued random variable $\beta(t)>0$ such that

$$
\left\|g_{t}(x)-g_{t}(y)\right\| \leq \beta(t)\|x(t)-y(t)\|
$$

for all $x(t), y(t) \in E$ and $t \in \Omega$.

Definition 2.10. Let $S: \Omega \times E \rightarrow E$ be a random operator. A operator $N: \Omega \times E \times E \times E \rightarrow E$ is said to be:

(a) Q-strongly accretive with respect to $S$ in the first argument if there exists $j_{2}(x(t)-$ $y(t)) \in J_{2}(x(t)-y(t))$ such that

$$
\left\langle N_{t}\left(S_{t}(x), \cdot, \cdot\right)-N_{t}\left(S_{t}(y), \cdot, \cdot\right), j_{2}(x(t)-y(t))\right\rangle \geq \rho(t)\|x(t)-y(t)\|^{2},
$$

for all $x(t), y(t) \in E$ and $t \in \Omega$, where $\rho(t)>0$ is a real-valued random variable;

(b) $\epsilon$-Lipschitz continuous in the first argument if there exists a real-valued random variable $\epsilon(t)>0$ such that

$$
\left\|N_{t}(x, \cdot, \cdot)-N_{t}(y, \cdot, \cdot)\right\| \leq \epsilon(t)\|x(t)-y(t)\|,
$$

for all $x(t), y(t) \in E$ and $t \in \Omega$.

Similarly, we can define the Lipschitz continuity in the second argument and third argument of $N(\cdot, \cdot, \cdot)$. 
Definition 2.11. Let $\eta: \Omega \times E \times E \rightarrow E^{*}$ be a random operator $H: \Omega \times E \rightarrow E$ be a random operator and $M: \Omega \times E \rightarrow 2^{E}$ be a random multivalued operator. Then $M$ is said to be:

(a) $\eta$-accretive if

$$
\left\langle u(t)-v(t), \eta_{t}(x, y)\right\rangle \geq 0
$$

for all $x(t), y(t) \in E, u(t) \in M_{t}(x)$ and $v(t) \in M_{t}(y)$ where $M_{t}(z)=M(t, z(t))$, for all $t \in \Omega$;

(b) strictly $\eta$-accretive if

$$
\left\langle u(t)-v(t), \eta_{t}(x, y)\right\rangle \geq 0
$$

for all $x(t), y(t) \in E, u(t) \in M_{t}(x), v(t) \in M_{t}(y)$ and $t \in \Omega$ and the equality holds if and only if $u(t)=v(t)$ for all $t \in \Omega$;

(c) $r$-strongly $\eta$-accretive if there exists a real-valued random variable $r(t)>0$ such that, for any $t \in \Omega$,

$$
\left\langle u(t)-v(t), \eta_{t}(x, y)\right\rangle \geq r(t)\|x(t)-y(t)\|^{2}
$$

for all $x(t), y(t) \in E, u(t) \in M_{t}(x), v(t) \in M_{t}(y)$ and $t \in \Omega$.

Definition 2.12. Let $\eta: \Omega \times E \times E \rightarrow E$ be a single-valued mapping, $A: \Omega \times E \rightarrow E$ be a single-valued mapping, $M: \Omega \times E \rightarrow 2^{E}$ be a multivalued mapping.

(i) $M_{t}$ is said to be m-accretive if $M_{t}$ is accretive and $(I+\rho(t) M(t, \cdot))(E)=E$ for all $t \in \Omega$ and $\rho(t)>0$, where $I$ is identity operator on $E$;

(ii) $M_{t}$ is said to be generalized m-accretive if $M_{t}$ is $\eta$-accretive and $(I+\rho(t) M(t, \cdot))(E)=E$ for all $t \in \Omega$ and $\rho(t)>0$;

(iii) $M_{t}$ is said to be $H_{t}$-accretive if $M_{t}$ is accretive and $\left(H_{t}+\rho(t) M(t, \cdot)\right)(E)=E$ for all $t \in \Omega$ and $\rho(t)>0$;

(iv) $M_{t}$ is said to be $\left(H_{t}, \eta\right)$-accretive if $M_{t}$ is $\eta$-accretive and $\left(H_{t}+\rho(t) M(t, \cdot)\right)(E)=E$ for all $t \in \Omega$ and $\rho(t)>0$.

Remark 2.13. If $E=E^{*}=H$ is a Hilbert space, then (a)-(c) of Definition 2.11 reduce to the definition of $\eta$-monotonicity, strict $\eta$-monotonicity and strong $\eta$-monotonicity, respectively, if $E$ is uniformly smooth and $\eta(x, y)=j_{2}(x-y) \in J_{2}(x-y)$, then (a)-(c) of Definition 2.11 reduce to the definitions of accretive, strictly accretive and strongly accretive in uniformly smooth Banach spaces, respectively.

Definition 2.14. The operator $\eta: \Omega \times E \times E \rightarrow E^{*}$ is said to be: $\tau$-Lipschitz continuous if there exists a real-valued random variable $\tau(t)>0$ such that

$$
\left\|\eta_{t}(x, y)\right\| \leq \tau(t)\|x(t)-y(t)\|
$$

for all $x(t), y(t) \in E$ and $t \in \Omega$. 
Definition 2.15. A multivalued measurable operator $T: \Omega \times E \rightarrow \mathrm{CB}(E)$ is said to be $\gamma-\widehat{H}$ Lipschitz continuous if there exists a measurable function $\gamma: \Omega \rightarrow(0,+\infty)$ such that, for any $t \in \Omega$,

$$
\widehat{H}\left(T_{t}(x), T_{t}(y)\right) \leq \gamma(t)\|x(t)-y(t)\|
$$

for all $x(t), y(t) \in E$.

Definition 2.16. Let $M: \Omega \times E \times E \rightarrow 2^{E}$ be a $\left(H_{t}, \eta\right)$-accretive random operator and $H$ : $\Omega \times E \rightarrow E$ be $r$-strongly monotone random operator. Then the proximal operator $J_{M_{t(, x)}}^{\rho(t), H_{t}}$ is defined as follows:

$$
J_{M_{t(, x)}}^{\rho(t), H_{t}}(z)=\left(H_{t}+\rho(t) M_{t}\right)^{-1}(z),
$$

for all $t \in \Omega$ and $z \in E$, where $\rho: \Omega \rightarrow(0, \infty)$ is a measurable function and $\eta: \Omega \times E \times E \rightarrow E^{*}$ is a strictly monotone operator.

Lemma 2.17 (see [31]). Let $\eta: E \times E \rightarrow E$ be a $\tau$-Lipschitz continuous operator, $H: \Omega \times E \rightarrow E$ be a r-strongly $\eta$-accretive operator and $M: \Omega \times E \rightarrow 2^{E}$ be an $\left(H_{t}, \eta\right)$-accretive operator. Then, the proximal operator $J_{M_{t}}^{\rho(t), H_{t}}: E \rightarrow E$ is $\tau^{q-1} / r$-Lipschitz continuous, that is,

$$
\left\|J_{M_{t}}^{\rho(t), H_{t}}(x)-J_{M_{t}}^{\rho(t), H_{t}}(y)\right\| \leq \frac{\tau^{q-1}}{r}\|x-y\|, \quad \forall x, y \in E, t \in \Omega
$$

\section{Random Iterative Algorithms}

In this section, we suggest and analyze a new class of iterative methods and construct some new random iterative algorithms for solving (2.7).

Lemma 3.1. The set of measurable mapping $x, u, v, w: \Omega \rightarrow E$ a random solution of problem (2.7) if and only if for all $t \in \Omega$, and

$$
p_{t}(x)=J_{M_{t(, x)}}^{\rho(t), H_{t}}\left(H_{t}\left(p_{t}(x)\right)-\rho(t) N_{t}\left(S_{t}(x), u, v\right)\right) .
$$

Proof. The proof directly follows from the definition of $J_{M_{t(, x)}}^{\rho(t), H_{t}}$ as follows:

$$
\begin{aligned}
p_{t}(x) & =J_{M_{t}(, w)}^{\rho(t), H_{t}}\left(H_{t}\left(p_{t}(x)\right)-\rho(t) N_{t}\left(S_{t}(x), u, v\right)\right) \\
& \Longleftrightarrow p_{t}(x)=\left(H_{t}+\rho(t) M_{t}\right)^{-1}\left[H_{t}\left(p_{t}(x)\right)-\rho(t) N_{t}\left(S_{t}(x), u, v\right)\right] \\
& \Longleftrightarrow H_{t}\left(p_{t}(x)\right)-\rho(t) N_{t}\left(S_{t}(x), u, v\right) \in H_{t}\left(p_{t}(x)\right)+\rho(t) M_{t}\left(p_{t}(x), w\right) \\
& \Longleftrightarrow 0 \in M_{t}\left(p_{t}(x), w\right)+N_{t}\left(S_{t}(x), u, v\right) .
\end{aligned}
$$


Algorithm 3.2. Suppose that $K, T, G: \Omega \times E \rightarrow \mathcal{F}(E)$ be three random fuzzy mappings satisfying the condition $(C)$. Let $\tilde{K}, \widetilde{T}, \tilde{G}: \Omega \times E \rightarrow \mathrm{CB}(E)$ be $\widehat{H}$-continuous random multivalued mappings induced by $K, T$, and $G$, respectively. Let $S, p: \Omega \times E \rightarrow E$, $\eta: \Omega \times E \times E \rightarrow E$ and $N: \Omega \times E \times E \times E \rightarrow E$ be random single-valued operators. Let $M: \Omega \times E \times E \rightarrow 2^{E}$ be a random multivalued operator such that for each fixed $t \in \Omega$ and $s \in E, M(t, \cdot, s): E \rightarrow 2^{E}$ be a $\left(H_{t}, \eta\right)$-accretive mapping and Range $(p) \cap \operatorname{dom} M(t, \cdot, s) \neq \emptyset$. For any given measurable mapping $x_{0}: \Omega \rightarrow E$, the multivalued mappings $\widetilde{K}\left(\cdot, x_{0}(\cdot)\right), \widetilde{T}\left(\cdot, x_{0}(\cdot)\right), \widetilde{G}\left(\cdot, x_{0}(\cdot)\right): \Omega \rightarrow E$ are measurable by Lemma 2.5. Then, there exists measurable selections $u_{0}(\cdot) \in \widetilde{K}\left(\cdot, x_{0}(\cdot)\right), v_{0}(\cdot) \in \widetilde{T}\left(\cdot, x_{0}(\cdot)\right)$ and $w_{0}(\cdot) \in \widetilde{G}\left(\cdot, x_{0}(\cdot)\right)$ by Himmelberg [6]. Set

$$
x_{1}(t)=x_{0}(t)-\lambda(t)\left\{p_{t}\left(x_{0}\right)-J_{M_{t}\left(\cdot, w_{0}\right)}^{\rho(t), H_{t}}\left[H_{t}\left(p_{t}\left(x_{0}\right)\right)-\rho(t) N_{t}\left(S_{t}\left(x_{0}\right), u_{0}, v_{0}\right)\right]\right\}
$$

where $\lambda(t) \rho(t)$ and $H_{t}$ are the same as in Lemma 3.1. Then it is easy to know that $x_{1}: \Omega \rightarrow E$ is measurable. Since $u_{0}(t) \in \widetilde{K}_{t}\left(x_{0}\right) \in \mathrm{CB}(E), v_{0}(t) \in \widetilde{T}_{t}\left(x_{0}\right) \in \mathrm{CB}(E)$ and $w_{0}(t) \in \widetilde{G}_{t}\left(x_{0}\right) \in$ $\mathrm{CB}(E)$, by Lemma 2.6, there exist measurable selections $u_{1}(t) \in \widetilde{K}_{t}\left(x_{1}\right), v_{1}(t) \in \widetilde{T}_{t}\left(x_{1}\right)$ and $w_{1}(t) \in \widetilde{G}_{t}\left(x_{1}\right)$ such that for all $t \in \Omega$,

$$
\begin{gathered}
\left\|u_{0}(t)-u_{1}(t)\right\| \leq\left(1+\frac{1}{1}\right) \widehat{H}\left(\widetilde{K}_{t}\left(x_{0}\right), \tilde{K}_{t}\left(x_{1}\right)\right), \\
\left\|v_{0}(t)-v_{1}(t)\right\| \leq\left(1+\frac{1}{1}\right) \widehat{H}\left(\widetilde{T}_{t}\left(x_{0}\right), \widetilde{T}_{t}\left(x_{1}\right)\right), \\
\left\|w_{0}(t)-w_{1}(t)\right\| \leq\left(1+\frac{1}{1}\right) \widehat{H}\left(\widetilde{G}_{t}\left(x_{0}\right), \widetilde{G}_{t}\left(x_{1}\right)\right) .
\end{gathered}
$$

By induction, we can define the sequences $\left\{x_{n}(t)\right\},\left\{u_{n}(t)\right\},\left\{v_{n}(t)\right\}$ and $\left\{w_{n}(t)\right\}$ inductively satisfying

$$
\begin{gathered}
x_{n+1}(t)=x_{n}(t)-\lambda(t)\left\{p_{t}\left(x_{n}\right)-J_{M_{t\left(;, w_{n}\right)}}^{\rho(t), H_{t}}\left[H_{t}\left(p_{t}\left(x_{n}\right)\right)-\rho(t) N_{t}\left(S_{t}\left(x_{n}\right), u_{n}, v_{n}\right)\right]\right\}, \\
u_{n}(t) \in \widetilde{M}_{t}\left(x_{n}\right), \quad\left\|u_{n}(t)-u_{n+1}(t)\right\| \leq\left(1+\frac{1}{n+1}\right) \widehat{H}\left(\widetilde{K}_{t}\left(x_{n}\right), \widetilde{K}_{t}\left(x_{n+1}\right)\right), \\
v_{n}(t) \in \widetilde{T}_{t}\left(x_{n}\right), \quad\left\|v_{n}(t)-v_{n+1}(t)\right\| \leq\left(1+\frac{1}{n+1}\right) \widehat{H}\left(\widetilde{T}_{t}\left(x_{n}\right), \widetilde{T}_{t}\left(x_{n+1}\right)\right), \\
w_{n}(t) \in \widetilde{G}_{t}\left(x_{n}\right), \quad\left\|w_{n}(t)-w_{n+1}(t)\right\| \leq\left(1+\frac{1}{n+1}\right) \widehat{H}\left(\widetilde{G}_{t}\left(x_{n}\right), \widetilde{G}_{t}\left(x_{n+1}\right)\right) .
\end{gathered}
$$

From Algorithm 3.2, we can get the following algorithms. 
Algorithm 3.3. Suppose that $E, M, \eta, S, \widetilde{K}, \tilde{T}$ and $\lambda$ are the same as in Algorithm 3.2. Let $\widetilde{G}: \Omega \times E \rightarrow E$ be a random single-valued operator, $p \equiv I$ and $N(t, x, y, z)=f(t, z)+g(t, x, y)$ for all $t \in \Omega$ and $x, y, z \in E$. Then, for given measurable $x_{0}: \Omega \rightarrow E$, we have

$$
\begin{aligned}
& x_{n+1}(t)=(1-\lambda(t)) x_{n}(t)+\lambda(t) J_{M_{t}\left(G_{t}\left(x_{n}\right)\right)}^{\rho(t), H_{t}}\left\{x_{n}(t)-\rho(t)\left[f_{t}\left(v_{n}\right)+g_{t}\left(S_{t}\left(x_{n}\right), u_{n}\right)\right]\right\}, \\
& u_{n}(t) \in \widetilde{K}_{t}\left(x_{n}\right), \quad\left\|u_{n}(t)-u_{n+1}(t)\right\| \leq\left(1+\frac{1}{n+1}\right) \widehat{H}\left(\widetilde{K}_{t}\left(x_{n}\right), \widetilde{K}_{t}\left(x_{n+1}\right)\right), \\
& v_{n}(t) \in \widetilde{T}_{t}\left(x_{n}\right), \quad\left\|v_{n}(t)-v_{n+1}(t)\right\| \leq\left(1+\frac{1}{n+1}\right) \widehat{H}\left(\widetilde{T}_{t}\left(x_{n}\right), \widetilde{T}_{t}\left(x_{n+1}\right)\right) .
\end{aligned}
$$

Algorithm 3.4. Let $M: \Omega \times E \rightarrow 2^{E}$ be a random multivalued operator such that for each fixed $t \in \Omega, M(t, \cdot): E \rightarrow 2^{E}$ is a $(H, \eta)$-accretive mapping and Range $(p) \bigcap \operatorname{dom} M(t, \cdot) \neq \emptyset$. If $S, p$, $\eta, N, \tilde{K}, \widetilde{T}$ and $\lambda$ are the same as in Algorithm 3.2, then for given measurable $x_{0}: \Omega \rightarrow E$, we have

$$
\begin{aligned}
& x_{n+1}(t)=x_{n}(t)-\lambda(t)\left\{p_{t}\left(x_{n}\right)-J_{M_{t\left(,, w_{n}\right)}^{\rho(t), H_{t}}}^{\rho}\left[p_{t}\left(x_{n}\right)-\rho(t) N_{t}\left(S_{t}\left(x_{n}\right), u_{n}, v_{n}\right)\right]\right\}, \\
& u_{n}(t) \in \widetilde{K}_{t}\left(x_{n}\right), \quad\left\|u_{n}(t)-u_{n+1}(t)\right\| \leq\left(1+\frac{1}{n+1}\right) \widehat{H}\left(\widetilde{K}_{t}\left(x_{n}\right), \widetilde{K}_{t}\left(x_{n+1}\right)\right), \\
& v_{n}(t) \in \widetilde{T}_{t}\left(x_{n}\right), \quad\left\|v_{n}(t)-v_{n+1}(t)\right\| \leq\left(1+\frac{1}{n+1}\right) \widehat{H}\left(\widetilde{T}_{t}\left(x_{n}\right), \widetilde{T}_{t}\left(x_{n+1}\right)\right) .
\end{aligned}
$$

\section{Existence and Convergence Theorems}

In this section, we prove the existence and convergence theorems of the generalized random iterative algorithm for this nonlinear random equations with random fuzzy mappings in $q$ uniformly smooth Banach spaces.

Theorem 4.1. Suppose that $E$ is a q-uniformly smooth and separable real Banach space, $p: \Omega \times E \rightarrow$ $E$ is $\alpha$-strongly accretive and $\beta$-Lipschitz continuous, $\eta: \Omega \times E \times E \rightarrow E$ be $\tau$-Lipschitz continuous, $H: \Omega \times E \rightarrow E$ is r-strongly $\eta$-accretive and $\mu_{A}$-Lipschitz continuous and $M: \Omega \times E \times E \rightarrow 2^{E}$ is a random multivalued operator such that for each fixed $t \in \Omega$ and $s \in E, M(t, \cdot, s): E \rightarrow 2^{E}$ is a $\left(H_{t}, \eta\right)$-accretive mapping and Range $(p) \bigcap \operatorname{dom} M(t, \cdot, s) \neq \emptyset$. Let $S: \Omega \times E \rightarrow E$ be a $\sigma$-Lipschitz continuous random operator and $N: \Omega \times E \times E \times E \rightarrow E$ be $\epsilon$-Lipschitz continuous in the first argument, $\mu$-Lipschitz continuous in the second argument and $v$-Lipschitz continuous in the third argument, respectively. Let $K, T, G: \Omega \times E \rightarrow \mathcal{F}(E)$ be three random fuzzy mappings satisfying the condition $(C), \widetilde{K}, \widetilde{T}, \widetilde{G}: \Omega \times E \rightarrow C B(E)$ be three random multivalued mappings induced by the mappings $K, T, G$, respectively, $K, T$ and $G$ are $\widehat{H}$-Lipschitz continuous with constants $\mu_{\tilde{K}}(t), \mu_{\tilde{T}}(t)$ and $\mu_{\widetilde{G}}(t)$, respectively. If for each real-valued random variables $\rho(t)>0$ and $\pi(t)>0$ such that, for any $t \in \Omega, x, y, z \in E$,

$$
\left\|J_{M_{t(\cdot, x)}(t), H_{t}}^{(z)}-J_{M_{t(\cdot, y)}}^{\rho(t), H_{t}}(z)\right\| \leq \pi(t)\|x-y\|
$$


and the following conditions hold:

$$
\begin{gathered}
l(t)=\left(1-q \alpha(t)+c_{q} \beta(t)^{q}\right)^{1 / q}+\pi(t) \mu_{\widetilde{G}}(t)<1 \\
\mu_{A}(t) \beta(t)+\rho(t) \epsilon(t) \sigma(t)+\mu(t) \mu_{\widetilde{K}}(t)+v(t) \mu_{\widetilde{T}}(t)<\frac{r(t)(1-l(t))}{\tau(t)^{q-1}},
\end{gathered}
$$

where $c_{q}$ is the same as in Lemma 2.8 for any $t \in \Omega$. If there exist real-valued random variables $\lambda(t), \rho(t)>0$ then there exist $x^{*}(t) \in E, u^{*}(t) \in K_{t}\left(x^{*}\right), v^{*}(t) \in T_{t}\left(x^{*}\right)$ and $w^{*}(t) \in G_{t}\left(x^{*}\right)$ such that $\left(x^{*}(t), u^{*}(t), v^{*}(t), w^{*}(t)\right)$ is solution of (2.7) and

$$
x_{n}(t) \longrightarrow x^{*}(t), \quad u_{n}(t) \longrightarrow u^{*}(t), \quad v_{n}(t) \longrightarrow v^{*}(t), \quad w_{n}(t) \longrightarrow w^{*}(t)
$$

as $n \rightarrow \infty$, where $\left\{x_{n}(t)\right\},\left\{u_{n}(t)\right\},\left\{v_{n}(t)\right\}$ and $\left\{w_{n}(t)\right\}$ are iterative sequences generated by Algorithm 3.2.

Proof. From Algorithm 3.2, Lemma 2.17 and (4.1), we compute

$$
\begin{aligned}
& \left\|x_{n+1}(t)-x_{n}(t)\right\| \\
& =\| x_{n}(t)-\lambda(t)\left\{p_{t}\left(x_{n}\right)-J_{M_{t\left(;, w_{n}\right)}^{\rho(t), H_{t}}}\left[H_{t}\left(p_{t}\left(x_{n}\right)\right)-\rho(t) N_{t}\left(S_{t}\left(x_{n}\right), u_{n}, v_{n}\right)\right]\right\} \\
& -x_{n-1}(t)+\lambda(t)\left\{p_{t}\left(x_{n-1}\right)-J_{\left.M_{t(, w} w_{n-1}\right)}^{\rho(t), H_{t}}\left[H_{t}\left(p_{t}\left(x_{n-1}\right)\right)-\rho(t) N_{t}\left(S_{t}\left(x_{n-1}\right), u_{n-1}, v_{n-1}\right)\right]\right\} \| \\
& \leq\left\|x_{n}(t)-x_{n-1}(t)-\lambda(t)\left(p_{t}\left(x_{n}\right)-p_{t}\left(x_{n-1}\right)\right)\right\| \\
& +\lambda(t) \| J_{M_{t\left(, ; w_{n}\right)}^{\rho(t), H_{t}}}^{\rho}\left[H_{t}\left(p_{t}\left(x_{n}\right)\right)-\rho(t) N_{t}\left(S_{t}\left(x_{n}\right), u_{n}, v_{n}\right)\right]
\end{aligned}
$$

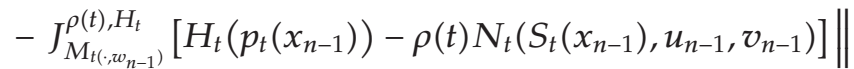

$$
\begin{aligned}
& \leq\left\|x_{n}(t)-x_{n-1}(t)-\lambda(t)\left(p_{t}\left(x_{n}\right)-p_{t}\left(x_{n-1}\right)\right)\right\| \\
& +\lambda(t) \| J_{M_{t\left(;, w_{n}\right)}^{\rho(t), H_{t}}}^{\rho}\left[H_{t}\left(p_{t}\left(x_{n}\right)\right)-\rho(t) N_{t}\left(S_{t}\left(x_{n}\right), u_{n}, v_{n}\right)\right] \\
& -J_{M_{t\left(;, w_{n}\right)}}^{\rho(t), H_{t}}\left[H_{t}\left(p_{t}\left(x_{n-1}\right)\right)-\rho(t) N_{t}\left(S_{t}\left(x_{n-1}\right), u_{n-1}, v_{n-1}\right)\right] \| \\
& \left.+\lambda(t) \| J_{M_{t\left(, ; w_{n}\right)}^{\rho(t), H_{t}}}^{\left(H_{t}\right.}\left(p_{t}\left(x_{n-1}\right)\right)-\rho(t) N_{t}\left(S_{t}\left(x_{n-1}\right), u_{n-1}, v_{n-1}\right)\right]
\end{aligned}
$$

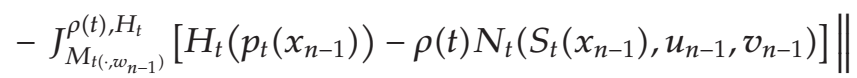

$$
\begin{aligned}
& \leq\left\|x_{n}(t)-x_{n-1}(t)-\lambda(t)\left(p_{t}\left(x_{n}\right)-p_{t}\left(x_{n-1}\right)\right)\right\| \\
& +\frac{\lambda(t) \tau(t)^{q-1}}{r(t)} \| H_{t}\left(p_{t}\left(x_{n}\right)\right)-\rho(t) N_{t}\left(S_{t}\left(x_{n}\right), u_{n}, v_{n}\right) \\
& \text { - }\left[H_{t}\left(p_{t}\left(x_{n-1}\right)\right)-\rho(t) N_{t}\left(S_{t}\left(x_{n-1}\right), u_{n-1}, v_{n-1}\right)\right] \| \\
& +\lambda(t) \pi(t)\left\|w_{n}-w_{n-1}\right\|
\end{aligned}
$$


Journal of Inequalities and Applications

$$
\begin{aligned}
& \leq(1-\lambda(t))\left\|x_{n}(t)-x_{n-1}(t)\right\|+\lambda(t)\left\|x_{n}(t)-x_{n-1}(t)-\left(p_{t}\left(x_{n}\right)-p_{t}\left(x_{n-1}\right)\right)\right\| \\
& +\frac{\lambda(t) \tau(t)^{q-1}}{r(t)}\{\| \\
& \quad\left(H_{t}\left(p_{t}\left(x_{n}\right)\right)-H_{t}\left(p_{t}\left(x_{n-1}\right)\right)\right) \| \\
& +\rho(t)\left\|\left(N_{t}\left(S_{t}\left(x_{n}\right), u_{n}, v_{n}\right)-N_{t}\left(S_{t}\left(x_{n-1}\right), u_{n}, v_{n}\right)\right)\right\| \\
& +\rho(t)\left\|N_{t}\left(S_{t}\left(x_{n-1}\right), u_{n}, v_{n}\right)-N_{t}\left(S_{t}\left(x_{n-1}\right), u_{n-1}, v_{n}\right)\right\| \\
& \left.\quad+\rho(t)\left\|N_{t}\left(S_{t}\left(x_{n-1}\right), u_{n-1}, v_{n}\right)-N_{t}\left(S_{t}\left(x_{n-1}\right), u_{n-1}, v_{n-1}\right)\right\|\right\} \\
& +\lambda(t) \pi(t)\left\|w_{n}-w_{n-1}\right\| .
\end{aligned}
$$

By using $p_{t}$ is a strongly accretive and Lipschitz continuous, we have

$$
\begin{aligned}
& \left\|x_{n}(t)-x_{n-1}(t)-\left(p_{t}\left(x_{n}\right)-p_{t}\left(x_{n-1}\right)\right)\right\|^{q} \\
& \leq\left\|x_{n}(t)-x_{n-1}(t)\right\|^{q}-q\left\langle\left(p_{t}\left(x_{n}\right)-p_{t}\left(x_{n-1}\right)\right), j_{q}\left(x_{n}(t)-x_{n-1}(t)\right)\right\rangle \\
& \quad \quad \quad c_{q}\left\|p_{t}\left(x_{n}\right)-p_{t}\left(x_{n-1}\right)\right\|^{q} \\
& \leq\left(1-q \alpha(t)+c_{q} \beta(t)^{q}\right)\left\|x_{n}(t)-x_{n-1}(t)\right\|^{q},
\end{aligned}
$$

that is

$$
\left\|x_{n}(t)-x_{n-1}(t)-\left(p_{t}\left(x_{n}\right)-p_{t}\left(x_{n-1}\right)\right)\right\| \leq\left(1-q \alpha(t)+c_{q} \beta(t)^{q}\right)^{1 / q}\left\|x_{n}(t)-x_{n-1}(t)\right\|,
$$

where $c_{q}$ is the same as in Lemma 2.8 .

By Lipschitz continuity of $N$ in the first, second and third argument, $S, p, H$ is $\sigma$ Lipschitz continuous, $\beta$-Lipschitz continuous, $\mu_{A}$-Lipschitz continuous, respectively, and $\widetilde{K}$, $\widetilde{T}$, and $\widetilde{G}$ are $H$-Lipschitz continuous, we have

$$
\begin{aligned}
& \left\|N_{t}\left(S_{t}\left(x_{n}\right), u_{n}, v_{n}\right)-N_{t}\left(S_{t}\left(x_{n-1}\right), u_{n}, v_{n}\right)\right\| \\
& \quad \leq \epsilon(t)\left\|S_{t}\left(x_{n}\right)-S_{t}\left(x_{n-1}\right)\right\| \\
& \quad \leq \epsilon(t) \sigma(t)\left\|x_{n}(t)-x_{n-1}(t)\right\|, \\
& \left\|N_{t}\left(S_{t}\left(x_{n-1}\right), u_{n}, v_{n}\right)-N_{t}\left(S_{t}\left(x_{n-1}\right), u_{n-1}, v_{n}\right)\right\| \\
& \quad \leq \mu(t)\left\|u_{n}-u_{n-1}\right\| \\
& \quad \leq \mu(t)\left(1+\frac{1}{n}\right) H\left(\widetilde{K}_{t}\left(x_{n-1}\right), \widetilde{K}_{t}\left(x_{n}\right)\right) \\
& \quad \leq\left(1+\frac{1}{n}\right) \mu(t) \mu_{\tilde{K}}(t)\left\|x_{n}(t)-x_{n-1}(t)\right\|,
\end{aligned}
$$




$$
\begin{aligned}
\| N_{t}( & \left.S_{t}\left(x_{n-1}\right), u_{n-1}, v_{n}\right)-N_{t}\left(S_{t}\left(x_{n-1}\right), u_{n-1}, v_{n-1}\right) \| \\
& \leq v(t)\left\|v_{n}-v_{n-1}\right\| \\
& \leq v(t)\left(1+\frac{1}{n}\right) H\left(\widetilde{T}_{t}\left(x_{n-1}\right), \widetilde{T}_{t}\left(x_{n}\right)\right) \\
& \leq\left(1+\frac{1}{n}\right) v(t) \mu_{\widetilde{T}}(t)\left\|x_{n}(t)-x_{n-1}(t)\right\|, \\
\|\left(H_{t}\right. & \left.\left(p_{t}\left(x_{n}\right)\right)-H_{t}\left(p_{t}\left(x_{n-1}\right)\right)\right) \| \\
& \leq \mu_{A}(t)\left\|p_{t}\left(x_{n}\right)-p_{t}\left(x_{n-1}\right)\right\| \\
& \leq \mu_{A}(t) \beta(t)\left\|x_{n}(t)-x_{n-1}(t)\right\|, \\
\| w_{n}- & w_{n-1} \| \\
& \leq\left(1+\frac{1}{n}\right) H\left(\widetilde{G}_{t}\left(x_{n-1}\right), \widetilde{G}_{t}\left(x_{n}\right)\right) \\
& \leq\left(1+\frac{1}{n}\right) \mu_{\widetilde{G}}(t)\left\|x_{n}(t)-x_{n-1}(t)\right\| .
\end{aligned}
$$

Using (4.6)-(4.7) in (4.4), we obtain for all $t \in \Omega$,

$$
\left\|x_{n+1}(t)-x_{n}(t)\right\| \leq \theta_{n}(t)\left\|x_{n}(t)-x_{n-1}(t)\right\|,
$$

where

$$
\begin{aligned}
\theta_{n}(t)= & 1-\lambda(t)+\lambda(t) \kappa_{n}(t), \\
\kappa_{n}(t)= & \left(1-q \alpha(t)+c_{q} \beta(t)^{q}\right)^{1 / q}+\left(1+\frac{1}{n}\right) \pi(t) \mu_{\widetilde{G}}(t) \\
& +\frac{\tau(t)^{q-1}}{r(t)}\left[\mu_{A}(t) \beta(t)+\rho(t) \epsilon(t) \sigma(t)+\rho(t)\left(1+\frac{1}{n}\right)\left(\mu(t) \mu_{\widetilde{K}}(t)+v(t) \mu_{\widetilde{T}}(t)\right)\right] .
\end{aligned}
$$

Letting

$$
\begin{aligned}
\mathcal{K}(t) & =\left(1-q \alpha(t)+c_{q} \beta(t)^{q}\right)^{1 / q}+\pi(t) \mu_{\tilde{G}}(t) \\
& +\frac{\tau(t)^{q-1}}{r(t)}\left[\mu_{A}(t) \beta(t)+\rho(t) \epsilon(t) \sigma(t)+\mu(t) \mu_{\tilde{K}}(t)+v(t) \mu_{\tilde{T}}(t)\right], \\
\theta(t) & =1-\lambda(t)+\lambda(t) \mathcal{\kappa}(t) .
\end{aligned}
$$

Thus $\kappa_{n}(t) \rightarrow \mathcal{k}(t), \theta_{n}(t) \rightarrow \theta(t)$ as $n \rightarrow \infty$. From (4.2), we know that $0<\theta(t)<1$, for all $t \in \Omega$. Using the same arguments as those used in the proof of (Lan et al. [11, Theorem 
3.1, page 14]) it follows that $\left\{x_{n}(t)\right\},\left\{u_{n}(t)\right\},\left\{v_{n}(t)\right\}$ and $\left\{w_{n}(t)\right\}$ are Cauchy sequences. Thus by the completeness of $E$, there exist $u^{*}(t), v^{*}(t), w^{*}(t) \in E$ such that $u_{n}(t) \rightarrow u^{*}(t), v_{n}(t) \rightarrow$ $v^{*}(t), w_{n}(t) \rightarrow w^{*}(t)$ as $n \rightarrow \infty$.

Next, we show that $u^{*}(t) \in K_{t}\left(x^{*}\right)$, we have

$$
\begin{aligned}
d\left(u^{*}(t), K_{t}\left(x^{*}\right)\right) & =\inf \left\{\left\|u^{*}(t)-y\right\|: y \in K_{t}\left(x^{*}\right)\right\} \\
& \leq\left\|u^{*}(t)-u_{n}(t)\right\|+d\left(u_{n}(t), K_{t}\left(x^{*}\right)\right) \\
& \leq\left\|u^{*}(t)-u_{n}(t)\right\|+H\left(K_{t}\left(x_{n}\right), K_{t}\left(x^{*}\right)\right) \\
& \leq\left\|u^{*}(t)-u_{n}(t)\right\|+\mu_{K}(t)\left\|x_{n}(t)-x^{*}(t)\right\| \longrightarrow 0, \quad n \longrightarrow \infty .
\end{aligned}
$$

This implies that $u^{*}(t) \in K_{t}\left(x^{*}\right)$. Similarly, we can get $v^{*}(t) \in T_{t}\left(x^{*}\right)$ and $w^{*}(t) \in G_{t}\left(x^{*}\right)$ for all $t \in \Omega$. Therefore, from Algorithm 3.2 and the continuity of $J_{M_{t(;), w)}}^{\rho(t), H_{t}}, p, N$ and $S$, we obtain

$$
p_{t}(x)=J_{\left.M_{t(;)}\right)}^{\rho(t), H_{t}}\left[H_{t}\left(p_{t}(x)\right)-\rho(t) N_{t}\left(S_{t}(x), u, v\right)\right] .
$$

By Lemma 3.1, we know that $\left(x^{*}(t), u^{*}(t), v^{*}(t), w^{*}(t)\right)$ is a solution of (2.7). This completes the proof.

Remark 4.2. If the fuzzy mapping $K, T$ and $G$ are multivalued operators, $H_{t} \equiv I$, and multivalued $M$ is generalized $m$-accretive mapping, $\eta$ is $\delta$-strongly monotone, $N$ is $Q^{-}$ strongly accretive with respect to $S$, then the result is Theorem 3.1 of Lan et al. [11].

From Theorem 4.1, we can get the following theorems.

Theorem 4.3. Let $E, \eta, S, H_{t}, \widetilde{K}, \widetilde{T}$ and $\lambda$ are the same as in Theorem 4.1. Assume that $M: \Omega \times E \times$ $E \rightarrow 2^{E}$ is a random multivalued operator such that, for each fixed $t \in \Omega$ and $s \in E, M(t, \cdot, s): E \rightarrow$ $2^{E}$ is a $\left(H_{t}, \eta\right)$-accretive mapping. Let $f: \Omega \times E \rightarrow E$ be $v$-Lipschitz continuous, $S: \Omega \times E \rightarrow E$ be a $\sigma$-Lipschitz continuous random operator, $\tilde{G}: \Omega \times E \rightarrow E$ be $\mu_{\tilde{G}}$-Lipschitz continuous and $g: \Omega \times E \times E \rightarrow E$ be $\epsilon$-Lipschitz continuous in the first argument and $\mu$-Lipschitz continuous in the second argument, respectively. If there exist real-valued random variables $\rho(t)>0$ and $\pi(t)>0$ such that (4.13) holds:

$$
1+\rho(t) \epsilon(t) \sigma(t)+\mu(t) \mu_{\tilde{K}}(t)+v(t) \mu_{\widetilde{T}}(t)<\frac{r(t)\left(1-\pi(t) \mu_{\widetilde{G}}(t)\right)}{\tau(t)^{q-1}}
$$

for all $t \in \Omega$, where $c_{q}$ is the same as in Lemma 2.8, for any $t \in \Omega$, the iterative sequences $\left\{x_{n}(t)\right\}$, $\left\{u_{n}(t)\right\}$ and $\left\{v_{n}(t)\right\}$ defined by Algorithm 3.3 converge strongly to the solution $\left(x^{*}(t), u^{*}(t), v^{*}(t)\right)$ of (2.8).

Theorem 4.4. Suppose that $E, p, S, \eta, N, M, T$ and $\lambda$ are the same as in Algorithm 3.2 Let $M$ : $\Omega \times E \rightarrow 2^{E}$ be a random multivalued operator such that, for each fixed $t \in \Omega, M(t, \cdot): E \rightarrow 2^{E}$ 
is a $\left(H_{t}, \eta\right)$-accretive mapping and Range $(p) \cap \operatorname{dom} M(t, \cdot) \neq \emptyset$. If there exists a real-valued random variable $\rho(t)>0$ such that, for any $t \in \Omega$, and the following conditions hold:

$$
\begin{gathered}
l(t)=\left(1-q \alpha(t)+c_{q} \beta(t)^{q}\right)^{1 / q}<1, \\
\mu_{A}(t) \beta(t)+\rho(t) \epsilon(t) \sigma(t)+\mu(t) \mu_{\widetilde{K}}(t)+v(t) \mu_{\tilde{T}}(t)<\frac{r(t)(1-l(t))}{\tau(t)^{q-1}},
\end{gathered}
$$

where $c_{q}$ is the same as in Lemma 2.8, for any $t \in \Omega$, the iterative sequences $\left\{x_{n}(t)\right\},\left\{u_{n}(t)\right\}$ and $\left\{v_{n}(t)\right\}$ defined by Algorithm 3.4 converge strongly to the solution $\left(x^{*}(t), u^{*}(t), v^{*}(t)\right)$ of $(2.9)$.

Remark 4.5. We note that for suitable choices of the mappings $S, p, H, M, K, T, G, \eta$ and space $E$. Theorems 4.1-4.4 reduces to many known results of generalized variational inclusions as special cases (see $[1,3,4,20-25,32]$ and the references therein).

\section{Acknowledgments}

This research is supported by the "Centre of Excellence in Mathematics", the Commission on High Education, Thailand. Moreover, N. Onjai-Uea is supported by the "Centre of Excellence in Mathematics", the Commission on High Education, Thailand for Ph.D. Program at King Mongkuts University of Technology Thonburi (KMUTT).

\section{References}

[1] R. P. Agarwal, Y. J. Cho, and N.-J. Huang, “Generalized nonlinear variational inclusions involving maximal $\eta$-monotone mappings," in Nonlinear Analysis and Applications: To V. Lakshmikantham on His 80th Birthday, vol. 1, 2, pp. 59-73, Kluwer Academic Publishers, Dordrecht, The Netherlands, 2003.

[2] R. P. Agarwal, M. F. Khan, D. O'Regan, and Salahuddin, "On generalized multivalued nonlinear variational-like inclusions with fuzzy mappings," Advances in Nonlinear Variational Inequalities, vol. 8, no. 1 , pp. 41-55, 2005.

[3] N.-J. Huang, "Generalized nonlinear variational inclusions with noncompact valued mappings," Applied Mathematics Letters, vol. 9, no. 3, pp. 25-29, 1996.

[4] N.-J. Huang and Y.-P. Fang, "A new class of general variational inclusions involving maximal $\eta$ monotone mappings," Publicationes Mathematicae Debrecen, vol. 62, no. 1-2, pp. 83-98, 2003.

[5] N.-J. Huang, Y. P. Fang, and C. X. Deng, "Nonlinear variational inclusions involving generalized $m$-accretive mappings," in Proceedings of the Bellman Continuum: International Workshop on Uncertain Systems and Soft Computing, pp. 323-327, Beijing, China, July 2002.

[6] C. J. Himmelberg, "Measurable relations," Fundamenta Mathematicae, vol. 87, pp. 53-72, 1975.

[7] N.-J. Huang, "Nonlinear implicit quasi-variational inclusions involving generalized $m$-accretive mappings," Archives of Inequalities and Applications, vol. 2, no. 4, pp. 413-425, 2004.

[8] H.-Y. Lan, "Projection iterative approximations for a new class of general random implicit quasivariational inequalities," Journal of Inequalities and Applications, vol. 2006, Article ID 81261, 17 pages, 2006.

[9] H.-Y. Lan, "Approximation solvability of nonlinear random $(A, \eta)$-resolvent operator equations with random relaxed cocoercive operators," Computers \& Mathematics with Applications, vol. 57, no. 4, pp. 624-632, 2009.

[10] H.-Y. Lan, "A class of nonlinear $(A, \eta)$-monotone operator inclusion problems with relaxed cocoercive mappings," Advances in Nonlinear Variational Inequalities, vol. 9, no. 2, pp. 1-11, 2006.

[11] H.-Y. Lan, Y. J. Cho, and W. Xie, "General nonlinear random equations with random multivalued operator in Banach spaces," Journal of Inequalities and Applications, vol. 2009, Article ID 865093, 17 pages, 2009. 
[12] M.-M. Jin and Q.-K. Liu, "Nonlinear quasi-variational inclusions involving generalized $m$-accretive mappings," Nonlinear Functional Analysis and Applications, vol. 9, no. 3, pp. 485-494, 2004.

[13] H. Y. Lan, J. K. Kim, and N.-J. Huang, "On the generalized nonlinear quasi-variational inclusions involving non-monotone set-valued mappings," Nonlinear Functional Analysis and Applications, vol. 9, no. 3, pp. 451-465, 2004.

[14] H.-Y. Lan, Q.-K. Liu, and J. Li, "Iterative approximation for a system of nonlinear variational inclusions involving generalized $m$-accretive mappings," Nonlinear Analysis Forum, vol. 9, no. 1, pp. 33-42, 2004.

[15] H.-Y. Lan, Z.-Q. He, and J. Li, "Generalized nonlinear fuzzy quasi-variational-like inclusions involving maximal $\eta$-monotone mappings," Nonlinear Analysis Forum, vol. 8, no. 1, pp. 43-54, 2003.

[16] L.-W. Liu and Y.-Q. Li, "On generalized set-valued variational inclusions," Journal of Mathematical Analysis and Applications, vol. 261, no. 1, pp. 231-240, 2001.

[17] R. U. Verma, M. F. Khan, and Salahuddin, "Generalized setvalued nonlinear mixed quasivariationallike inclusions with fuzzy mappings," Advances in Nonlinear Variational Inequalities, vol. 8, no. 2, pp. 11-37, 2005.

[18] R. Ahmad and F. F. Bazán, "An iterative algorithm for random generalized nonlinear mixed variational inclusions for random fuzzy mappings," Applied Mathematics and Computation, vol. 167, no. 2, pp. 1400-1411, 2005.

[19] S. S. Chang, Fixed Point Theory with Applications, Chongqing, Chongqing, China, 1984.

[20] S. S. Chang, Variational Inequality and Complementarity Problem Theory with Applications, Shanghai Scientific and Technological Literature, Shanghai, China, 1991.

[21] S. S. Chang and N.-J. Huang, "Generalized random multivalued quasi-complementarity problems," Indian Journal of Mathematics, vol. 35, no. 3, pp. 305-320, 1993.

[22] Y. J. Cho, S. H. Shim, N.-J. Huang, and S. M. Kang, "Generalized strongly nonlinear implicit quasivariational inequalities for fuzzy mappings," in Set Valued Mappings with Applications in Nonlinear Analysis, vol. 4 of Mathematical Analysis and Applications, pp. 63-77, Taylor \& Francis, London, UK, 2002.

[23] Y. J. Cho, N.-J. Huang, and S. M. Kang, "Random generalized set-valued strongly nonlinear implicit quasi-variational inequalities," Journal of Inequalities and Applications, vol. 5, no. 5, pp. 515-531, 2000.

[24] Y. J. Cho and H.-Y. Lan, "Generalized nonlinear random $(A, \eta)$-accretive equations with random relaxed cocoercive mappings in Banach spaces," Computers \& Mathematics with Applications, vol. 55, no. 9, pp. 2173-2182, 2008.

[25] H. R. Feng and X. P. Ding, "A new system of generalized nonlinear quasi-variational-like inclusions with A-monotone operators in Banach spaces," Journal of Computational and Applied Mathematics, vol. 225, no. 2, pp. 365-373, 2009.

[26] N.-J. Huang and Y. J. Cho, "Random completely generalized set-valued implicit quasi-variational inequalities," Positivity, vol. 3, no. 3, pp. 201-213, 1999.

[27] N.-J. Huang, X. Long, and Y. J. Cho, "Random completely generalized nonlinear variational inclusions with non-compact valued random mappings," Bulletin of the Korean Mathematical Society, vol. 34, no. 4, pp. 603-615, 1997.

[28] M. A. Noor and S. A. Elsanousi, "Iterative algorithms for random variational inequalities," Panamerican Mathematical Journal, vol. 3, no. 1, pp. 39-50, 1993.

[29] R. U. Verma, "A class of projection-contraction methods applied to monotone variational inequalities," Applied Mathematics Letters, vol. 13, no. 8, pp. 55-62, 2000.

[30] H. K. Xu, "Inequalities in Banach spaces with applications," Nonlinear Analysis: Theory, Methods $\mathcal{E}$ Applications, vol. 16, no. 12, pp. 1127-1138, 1991.

[31] R. Ahmad and A. P. Farajzadeh, "On random variational inclusions with random fuzzy mappings and random relaxed cocoercive mappings," Fuzzy Sets and Systems, vol. 160, no. 21, pp. 3166-3174, 2009.

[32] N.-J. Huang, "Random generalized nonlinear variational inclusions for random fuzzy mappings," Fuzzy Sets and Systems, vol. 105, no. 3, pp. 437-444, 1999. 